Article

\title{
Immobilization of Poly(1,1-dimethysilacyclobutane) by Means of Anionic Ring-Opening Polymerization on Organic Nanoparticles and Reinvestigation of Crystallization
}

\author{
Markus Gallei $^{1{ }^{*} \text {, Junyu Li }}{ }^{2}$, Johannes Elbert ${ }^{1}$, Markus Mazurowski ${ }^{1}$, Astrid Schönberger ${ }^{1}$,
} Christian Schmidt ${ }^{1}$, Bernd Stühn ${ }^{2, *}$ and Matthias Rehahn ${ }^{1}$

1 Ernst-Berl Institute for Chemical Engineering and Macromolecular Science,

Darmstadt University of Technology, Petersenstraße 22, D-64287 Darmstadt, Germany;

E-Mails: j.elbert@mc.tu-darmstadt.de (J.E.); m.mazurowski@mc.tu-darmstadt.de (M.M.);

a.schoenberger@mc.tu-darmstadt.de (A.S.); c.schmidt@mc.tu-darmstadt.de (C.S.);

m.rehahn@mc.tu-darmstadt.de (M.R.)

2 Institute of Condensed Matter Physics, Darmstadt University of Technology, Hochschulstraße 8, D-64289 Darmstadt, Germany; E-Mail: 1ijy@iccas.ac.cn

* Author to whom correspondence should be addressed;

E-Mails: m.gallei@mc.tu-darmstadt.de (M.G.); stuehn@fkp.physik.tu-darmstadt.de (B.S.);

Tel.: +49-6151-164545(M.G.); Fax: +49-6151-164670 (M.G.).

Received: 30 January 2013; in revised form: 28 February 2013 / Accepted: 5 March 2013 /

Published: 15 March 2013

Abstract: In the present study, the synthesis of poly(1,1-dimethylsilacyclobutane) (PDMSB) by anionic ring opening polymerization (ROP) is reinvestigated, leading to narrowly distributed molar masses (polydispersities 1.04-1.15) in the range of 2.3 to $60 \mathrm{~kg} \mathrm{~mol}^{-1}$. Investigations of thermal behavior for low molar mass PDMSB revealed an untypical multiple peaks melting phenomenon, which at first glance, seems to be of the same origin as low molar mass poly(ethylene oxide)s. Small angle X-ray scattering (SAXS) and X-ray diffraction (XRD) measurements are done, proving the fast crystallization and subsequent recrystallization for investigated low molar mass samples. Synthetic attempts are expanded to the surface-initiated anionic ROP of 1,1-dimethylsilacyclobutane (DMSB) monomer from the surface of cross-linked polystyrene (PS) nanoparticles. Novel polycarbosilanes (PCS)/organic core/shell particles are obtained, which are investigated by using transmission electron microscopy (TEM) and dynamic light scattering (DLS) experiments. First insights into the crystallization behavior 
of surface-attached PDMSB chains reveal that crystallization seems to be hindered.

Keywords: polycarbosilanes; anionic polymerizations; crystallization; nanoparticles; surface-attached polymers

\section{Introduction}

Polycarbosilanes (PCS) gained considerable attention in the last two decades, due to their interesting optoelectronic and heat-resistant applications, but most important are applications as oxygen-free ceramic precursors [1-5]. Recently, ordered mesoporous non-oxide materials [6], silicon carbide fibers [7] and ceramic foams [8] could be obtained by the so-called polymer precursor route of PCS and metal-substituted PCS derivatives after pyrolysis [9]. There are various synthetic routes for controlling the PCS structure, which is unambiguously important for pursued ceramic materials with sufficient ceramic yields. Strategies range from Kumada rearrangement of silanes [10], Grignard coupling [11-13] to $\mathrm{Pt}(0)$-catalyzed ring opening polymerization (ROP) of cyclic carbosilanes [9,14]. The latter method has the advantage that well-defined hyperbranched materials can be obtained with high ceramic yields after pyrolysis. A postmodification of more intensively investigated polymers, e.g., polyisoprene or poly(ethylene oxide) (PEO) and also unsaturated PCS via hydrosilylation is a feasible strategy to introduce pendant side groups with additional functionalities [15-20]. The above mentioned synthetic strategies suffer from the disadvantages that well-defined PCS with respect to narrowly distributed high molar masses cannot be obtained, as well as it is impossible to gain block copolymer structures. Amongst all synthetic protocols, anionic polymerization - especially anionic ROP - has several advantages and enables one to synthesize various block copolymers (BC) with precise control over block compositions, a well-defined constitution and narrowly distributed molar masses. Fascinating microstructures for manifold applications are accessible via self-assembly of such polymers [21]. For example, well-defined BCs consisting of polyisoprene and polymers with laterally bonded alkyl-silane groups were recently prepared by sequential anionic polymerization and have been used as membranes for gas separation applications [22]. The anionic ring opening polymerization (ROP) of ring-strained cyclic silanes, carbosilanes and metal-containing carbosilanes has also been reported and lead to well-defined polymers [23-32]. Out of all cyclic carbosilanes, 1,1-dimethylsilacyclobutane (DMSB) is a well-known monomer, due to its convenient chemical and also commercial availability. The anionic ROP of DMSB leads to constitutional well-defined crystallizable polymers. Furthermore, it is reported that polymerization in a polar solvent, such as tetrahydrofuran (THF), at low temperature forces results in poly(1,1-dimethylsilacyclobutane) (PDMSB) chains precipitating during the polymerization and, hence, a loss of the "living" nature of the chains occurs, hampering the possibility of BC formation [33,34]. In spite of those obstacles, PDMSB-containing block copolymers with polystyrene (PS) have been synthesized by Kim et al. using a slightly modified synthetic protocol [35]. Fascinating BC structures with PS have been obtained after BC self-assembly, and even after subsequent pyrolysis, highly-ordered ceramic structures could be maintained. The synthesis and microphase separation of BCs consisting of PDMSB and various ferrocene-containing polymers have also been reported [36]. In the present study, we 
firstly synthesize and investigate PDMSB homopolymers obtained by anionic ROP, and results are compared to previously reported studies. Absolute molar masses, which are determined via multi-angle laser light scattering size exclusion chromatography (SEC-MALLS) measurements, are varied in the range of $2.3 \mathrm{~kg} \mathrm{~mol}^{-1}$ to $60 \mathrm{~kg} \mathrm{~mol}^{-1}$, with low polydispersities for every sample (polydispersity index (PDI) values range from 1.04 to 1.15). The thermal behavior of PDMSB is reinvestigated by differential scanning calorimetry (DSC) measurements revealing two melting peaks, but only one crystallization peak for PDMSB chains with low molar masses. Prima facie, this crystallization behavior seems similar to studies with poly(ethylene oxide)s [37]. Small angle X-ray scattering (SAXS) and X-ray diffraction (XRD) are applied to ascertain whether this untypical crystallization behavior is based on two different crystal forms of PDMSB or caused by lamellar thickening of the investigated samples. PDMSB seems to be an excellent candidate for studying the crystallization of anchored polymers influenced on a curved substrate, due to its distinct and rapid crystallization. Thus, our attempts are expanded to the surface-initiated anionic ROP on the surface of cross-linked PS nanoparticles to gain PCS/organic core/shell particles. The PCS-grafted particles are characterized by using transmission electron microscopy (TEM), while dynamic light scattering (DLS) is applied to study the hydrodynamic radii of novel core/shell particles in dispersion. Additionally, the crystallization of surface-attached PDMSB is investigated via DSC measurements and results compared with free PDMSB chains. We emphasize that the herein described physical characterization leads to a more profound understanding of PDMSB, which behaves different with respect to crystallization compared to other crystallizable polymers with low glass transition temperatures, such as poly(ethylene oxide) (PEO). The synthetic protocol for surface-initiated anionic ROP of DMSB leads to novel well-defined PCS/organic core/shell structures, which are promising candidates to gain materials for SiC-based ceramic particles or hollow spheres after pyrolysis. Such $\mathrm{SiC}$ materials find important applications for electric devices, such as sensors or in catalysis [38-40].

\section{Experimental Section}

\subsection{Instrumentation}

Standard SEC was performed with THF as the mobile phase (flow rate $1 \mathrm{~mL} \mathrm{~min}^{-1}$ ) on a SDV column set from PSS (SDV 1000, SDV 100000, SDV 1000000) at $30^{\circ} \mathrm{C}$. Calibration was carried out using PS standards (from Polymer Standard Service, Mainz). For the SEC-MALLS experiments, a system composed of a Waters 515 pump (Waters, Milford, CT), a TSP AS100 autosampler, a Waters column oven, a Waters 486 UV detector operating at $254 \mathrm{~nm}$, a Waters 410 RI-detector and a DAWN DSP light scattering detector (Wyatt Technology, Santa Barbara, CA) was used. For data acquisition and evaluation of the light-scattering experiments, Astra version 4.73 (Wyatt Technology, Santa Barbara, CA) was used. The light-scattering instrument was calibrated using pure toluene, assuming a Rayleigh ratio of $9.78 \times 10^{-6} \mathrm{~cm}^{-1}$ at $690 \mathrm{~nm}$. An injection volume of $118 \mu \mathrm{L}$, a sample concentration of 1-2 $\mathrm{g} \mathrm{L}^{-1}$, a column temperature of $35{ }^{\circ} \mathrm{C}$ and a THF flow rate of $1 \mathrm{~mL} \mathrm{~min}^{-1}$ have been applied. SEC analysis was performed on a high resolution column set from PSS (SDV $5 \mu \mathrm{m} 106 \AA$, SDV $5 \mu \mathrm{m}$ $105 \AA$, SDV $5 \mu \mathrm{m} 1000 \AA$ ). Subsequent slow addition of monomer for the PS latex synthesis was carried out by using a precision piston pump (mikro Prominent). TEM experiments were carried out 
with a Zeiss EM 10 electron microscope operating at $80 \mathrm{kV}$. All shown images were recorded with a slow-scan charge-coupled device (CCD) camera obtained from TRS (Tröndle) in bright field mode. Camera control was computer aided using the ImageSP software from TRS. Centrifugation was performed with an Avanti J-30I from Beckman Coulter for half an hour with a speed of 17,000 rpm. Dynamic light scattering (DLS) experiments were performed with a setup based on a He-Ne laser with a wavelength of $\lambda=632.8 \mathrm{~nm}$ and a goniometer allowing one to measure at scattering angles $2 \theta$ from $30^{\circ}$ to $160^{\circ}$. Polarization of the primary beam was defined by a Glan-Thomson prism. The scattered beam polarization was again analyzed for its polarization, and all experiments were performed in vertical-vertical geometry. Scattered intensity was detected with an optical fiber coupled to two phototubes. In this way, background could be minimized using the cross-correlated signal from both tubes. The correlation function of intensity was calculated with an ALV 5000 autocorrelator. The sample was filled in a cylindrical cuvette (Hellma) and immersed in a temperature controlled index matching bath. DLS measurements were carried out at $25^{\circ} \mathrm{C}$ in THF. The concentrations of the nanoparticle dispersions were approximately $0.01 \mathrm{w} \%$ after ultrasonic treatment $(3 \mathrm{~h}$ for the polymer-grafted particles and $6 \mathrm{~h}$ for bare PS particles). After that sonication time, the experimentally obtained value of the hydrodynamic radius, $R_{h}$, was constant. The scattering intensity was measured in a range of scattering angles, $\theta$, from $50^{\circ}$ to $130^{\circ}$. The autocorrelation function of intensity, $\mathrm{g}_{2}(\mathrm{t}, q)$, was thus obtained. SAXS measurements were carried using a custom made system. The scattered intensity was measured as a function of scattering vector $q=4 \pi / \lambda \sin \theta$, where $\lambda$ is the X-ray wavelength and $2 \theta$ the scattering angle. The experimental setup used monochromatized $\mathrm{Cu} \mathrm{K}$ radiation with $\lambda=1.54 \AA$, a pinhole collimation and a two-dimensional position sensitive detector by Molecular Metrology. The accessed q-range is from $0.008 \AA^{-1}$ to $0.25 \AA^{-1}$. The two-dimensional scattering image from the detector was calibrated using Ag-behenate and radially averaged to calculate the intensity $\mathrm{I}(q)$. Samples were measured in borosilicate capillaries with a $1.5 \mathrm{~mm}$ diameter, and temperature was controlled with an accuracy of $\Delta \mathrm{T}=0.2 \mathrm{~K}$. XRD was conducted using a Siemens D500 diffractometer in the reflection mode using an incident X-ray wavelength of $1.54 \AA$ with a scan rate of $0.04^{\circ} \min ^{-1}$. The diffractometer was equipped with a hot stage to control the temperature of the sample with an accuracy of $\Delta \mathrm{T}=1 \mathrm{~K}$. The resulting plots of X-ray intensity versus $2 \theta$ were analyzed using the profile fitting program. DSC measurements were done on a computer aided TA Instruments DSC Q1000. The sample mass was in the range of 2-4 mg for PDMSB homopolymer, while for PDMSB-grafted PS particles, the mass used was in the range of 12 to $15 \mathrm{mg}$. Dried $\mathrm{N}_{2}$ gas was purged with a constant flow rate during the measurement. The temperature reading and caloric measurements were calibrated using indium and blue sapphire as the standard.

\subsection{Materials}

All solvents and reagents were purchased from Alfa Aesar, Sigma Aldrich, Fisher Scientific and ABCR and used as received, unless otherwise stated. Tetrahydro-furan (THF), toluene and $n$-hexane were distilled from sodium/benzophenone under reduced pressure (cryo-transfer) prior to the addition of 1,1-diphenylethylene and $n$-butyllithium ( $n$-BuLi), followed by a second cryo-transfer. 1,1-dimethysilacyclobutane (DMSB) was dried over $\mathrm{CaH}_{2}$ and distilled, followed by the addition of 1,1-diphenylethylenelithium (DPHLi), until the red color remained. DMSB was freshly distilled from 
that solution prior to the anionic ROP. All syntheses were carried out under an atmosphere of nitrogen or argon using the Schlenk technique or a glovebox equipped with a Coldwell apparatus.

\subsection{Anionic Polymerization of 1,1-Dimethylsilacyclobutane (DMSB)}

Exemplary synthesis for PDMSB with a molar mass of Approx. $30 \mathrm{~kg} \mathrm{~mol}^{-1}$ : In a glovebox, $470 \mathrm{mg}$ DMSB (4.69 mmol) is dissolved in $20 \mathrm{~mL}$ of neat THF in an ampoule equipped with a stirring bar. The solution is cooled to $-60{ }^{\circ} \mathrm{C}$ by using the Coldwell apparatus, and the polymerization is initiated by adding $7 \mu \mathrm{L} n$-BuLi $(1.6 \mathrm{M}, 11.2 \mu \mathrm{mol})$. After $1 \mathrm{~h}$, the reaction is terminated with methanol and the solution poured into a 10-fold excess of methanol. The polymer precipitates as a colorless solid, is filtered and dried in vacuo (yield: $454 \mathrm{mg}$, 97\%). Molar mass is determined by using SEC-MALLS $\left(\mathrm{M}_{\mathrm{n}}=31,500, \mathrm{M}_{\mathrm{w}}=32,600, \mathrm{PDI}=1.04\right)$.

\subsection{Synthesis of Cross-Linked PS Particles}

In a $500 \mathrm{~mL}$ three-necked flask equipped with magnetic stirring bar, $105 \mathrm{~mL}$ degassed water, $6.30 \mathrm{~g}$ styrene, $0.70 \mathrm{~g}$ divinylbenzene and $0.1 \mathrm{~g}$ sodium dodecylsulfate are added. The mixture is stirred and heated to $75^{\circ} \mathrm{C}$. A solution of $0.175 \mathrm{~g}$ sodium peroxodisulfate and $0.035 \mathrm{~g}$ sodium dithionite in $5 \mathrm{~mL}$ water are added to initiate the batch polymerization. After $30 \mathrm{~min}$, an emulsion of $37.8 \mathrm{~g}$ styrene, $4.2 \mathrm{~g}$ divinylbenzene, $0.35 \mathrm{~g}$ sodium dodecylsulfate and $0.42 \mathrm{~g}$ Triton X405 in $86 \mathrm{~mL}$ degassed water is added continuously with a precision piston pump (flow rate of $1 \mathrm{~mL} \mathrm{~min}^{-1}$ ). After the addition, stirring is continued for $1 \mathrm{~h}$ at $75^{\circ} \mathrm{C}$ with a stirring rate of $300 \mathrm{rpm}$. The resulting PS latex was characterized by using TEM, revealing a particle diameter of approximately $230 \mathrm{~nm}$.

\subsection{Surface-Initiated Anionic Ring Opening Polymerization of DMSB}

PDMSB@PS 1: In an ampoule equipped with a stirring bar, $300 \mathrm{mg}$ of dried cross-linked PS particles are dispersed with $7.5 \mathrm{~mL}$ dry toluene by stirring vigorously for $24 \mathrm{~h}$ inside a glovebox. $45 \mu \mathrm{L} \mathrm{sec}-\mathrm{BuLi}(\mathrm{c}=1.3 \mathrm{M}, 35 \mu \mathrm{mol})$ are added, and the dispersion turns slightly orange. After stirring the dispersion for $1 \mathrm{~h}$ at room temperature, it is transferred into an ampoule with cooled $\mathrm{THF}\left(-60{ }^{\circ} \mathrm{C}\right)$. After stirring the dispersion for $30 \mathrm{~min}$ cooled inside the Coldwell apparatus at $-60{ }^{\circ} \mathrm{C}, 280 \mathrm{mg}$ precooled DMSB $\left(-15^{\circ} \mathrm{C}\right)$ are added. The solution is stirred for $2.5 \mathrm{~h}$ at $-60{ }^{\circ} \mathrm{C}$. The reaction is terminated with methanol and the particle dispersion centrifuged for $30 \mathrm{~min}$ at 15,000 rpm. To get rid of impurities and freely formed PDMSB chains, the particles are dispersed in toluene and again centrifuged. This step is repeated at least five times, followed by drying of the functionalized particles in vacuo.

PDMSB@,PS 2: In an ampoule equipped with a stirring bar, $280 \mathrm{mg}$ of dried cross-linked PS particles are dispersed with $7.5 \mathrm{~mL}$ dry toluene by stirring vigorously for $24 \mathrm{~h}$ inside a glovebox. $20 \mu \mathrm{L} \mathrm{sec}$-BuLi (c = 1.3 M, $15 \mu \mathrm{mol})$ are added, and the dispersion turns slightly orange. After stirring the dispersion for $1 \mathrm{~h}$ at room temperature, it is transferred into an ampoule with cooled $\mathrm{THF}\left(-60^{\circ} \mathrm{C}\right)$. After stirring the dispersion for $30 \mathrm{~min}$ at $-60{ }^{\circ} \mathrm{C}$ inside the Coldwell apparatus, $280 \mathrm{mg}$ precooled DMSB $\left(-15^{\circ} \mathrm{C}\right)$ are added. The solution is stirred for $2.5 \mathrm{~h}$ at $-60{ }^{\circ} \mathrm{C}$. The reaction is terminated with methanol and the particle dispersion centrifuged for $30 \mathrm{~min}$ at 15,000 rpm. To get rid of impurities and 
freely formed PDMSB chains, the particles are dispersed in toluene and again centrifuged. This step is repeated at least five times, followed by drying of the functionalized particles in vacuo.

\section{Results and Discussion}

\subsection{PDMSB Synthesis}

Although anionic ROP of DMSB monomer in THF at low temperatures $\left(-48{ }^{\circ} \mathrm{C}\right.$ to $\left.-60{ }^{\circ} \mathrm{C}\right)$ (Figure 1) leads to a turbidity after approximately 10 to $15 \mathrm{~min}$ after addition of $n$-BuLi as initiator independently of the intended molar mass, high molar masses up to $60 \mathrm{~kg} \mathrm{~mol}^{-1}$ could be obtained with low polydispersity values (PDI < 1.15). The results are summarized in Table 1.

Figure 1. Anionic ring opening polymerization (ROP) of 1,1-dimethysilacyclobutane (DMSB).

$\mathrm{n}$<smiles>C[Si]1(C)CCC1</smiles>

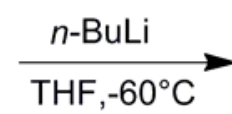

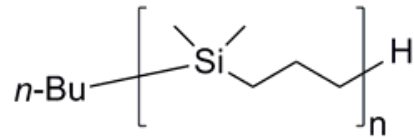

Table 1. Size exclusion chromatography (SEC) with polystyrene (PS) standards vs. multi-angle laser light scattering size exclusion chromatography (SEC-MALLS) results of investigated PDMSB homopolymers.

\begin{tabular}{cccccc}
\hline $\mathbf{M}_{\mathbf{n}}{ }^{\mathbf{a}}\left(\mathbf{g ~ m o l}^{-\mathbf{1}}\right)$ & $\mathbf{M}_{\mathbf{w}}{ }^{\mathbf{a}}\left(\mathbf{g ~ m o l}^{-\mathbf{1}}\right)$ & $\mathbf{P D I}^{\mathbf{a}}\left(\mathbf{g ~ m o l}^{\mathbf{- 1}}\right)$ & $\mathbf{M}_{\mathbf{n}}{ }^{\mathbf{b}}\left(\mathbf{g ~ m o l}^{-\mathbf{1}}\right)$ & $\mathbf{M}_{\mathbf{w}}{ }^{\mathbf{b}}\left(\mathbf{g ~ m o l}^{-1}\right)$ & $\mathbf{P D I}^{\mathbf{b}}$ \\
\hline 2250 & 2590 & 1.15 & 4110 & 4650 & 1.13 \\
5200 & 5620 & 1.08 & 7220 & 8190 & 1.13 \\
8210 & 8920 & 1.09 & 9920 & 10480 & 1.06 \\
28680 & 32900 & 1.15 & 31450 & 32610 & 1.04 \\
52250 & 59760 & 1.14 & 57590 & 59700 & 1.04 \\
\hline
\end{tabular}

${ }^{a}$ Molecular weight and PDI obtained by SEC measurements (PS standards, THF); ${ }^{b}$ molecular weight and PDI obtained by SEC-MALLS measurements; the refractive index increment $\mathrm{dn} / \mathrm{dc}$ could be determinated to 0.0966 (THF, $30^{\circ} \mathrm{C}$ ). THF: tetrahydrofuran; PDI: polydispersity index.

From Table 1, it becomes clear that molar masses obtained via conventional SEC with PS standards were slightly lower compared to the absolute molar mass values obtained by using SEC-MALLS. An exemplary monomodal, narrowly distributed SEC-MALLS trace for PDMSB with an average molar mass of approximately $32 \mathrm{~kg} \mathrm{~mol}^{-1}$ is given in Figure 2. The anionic ROP of DMSB is quite fast, and living chain ends can be thought to be very reactive. However, after a longer reaction time $(>1 \mathrm{~h})$ under applied conditions, PDMSB samples showed a significant shoulder in SEC traces shifted to higher molar masses, which can be assumed to be caused by an undesired coupling process of living chain ends with the silane moieties. 
Figure 2. Exemplary SEC-MALLS trace (a) and molar mass distribution (b) of PDMSB obtained by SEC-MALLS measurement synthesized via anionic ROP in THF at $-60{ }^{\circ} \mathrm{C}$.

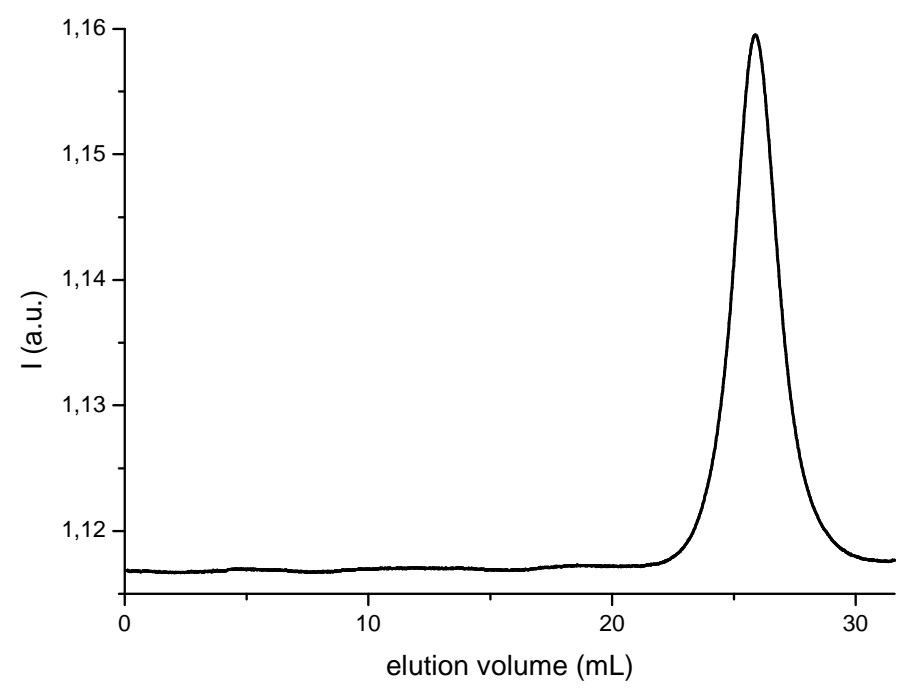

a

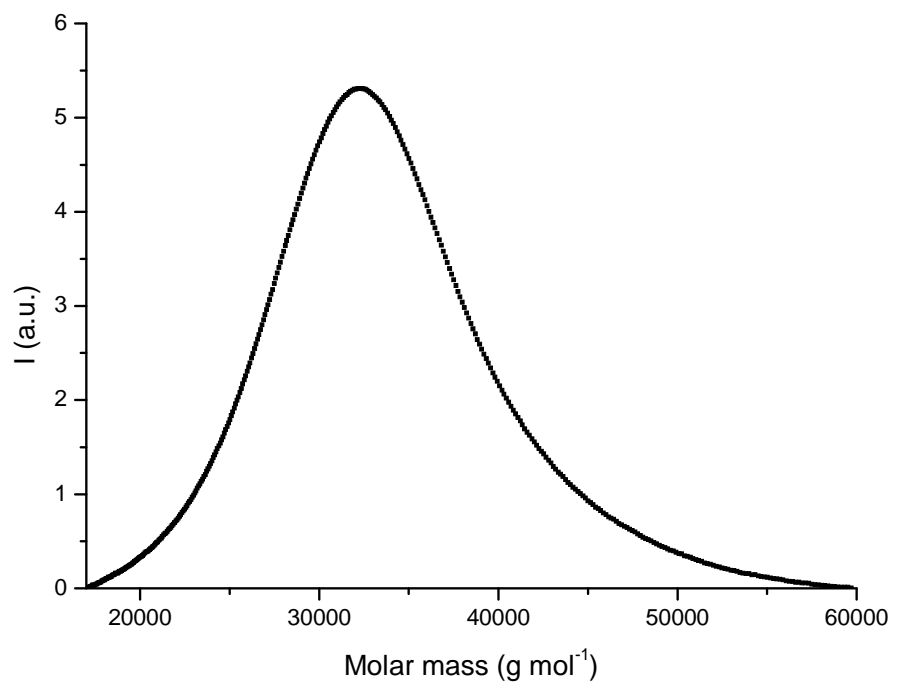

b

\subsection{DSC Measurements of PDMSB Homopolymers}

For a study of the melting and crystallization behavior of PDMSB homopolymers, samples were heated with a rate of $10 \mathrm{~K} \mathrm{~min}^{-1}$ to $353 \mathrm{~K}$, and the temperature was kept constant for 10 min to delete thermal history. Then, the sample was cooled to $253 \mathrm{~K}$ with a rate of $10 \mathrm{~K} \mathrm{~min}^{-1}$ to obtain the DSC exotherms, followed by heating again to obtain the DSC endotherms. In Figure 3, the DSC curve of PDMSB with an average molar mass of $5.6 \mathrm{~kg} \mathrm{~mol}^{-1}$ is exemplarily given. 
Figure 3. Differential scanning calorimetry (DSC) diagram of PDMSB with an average molar mass of $5.6 \mathrm{~kg} \mathrm{~mol}^{-1}$.

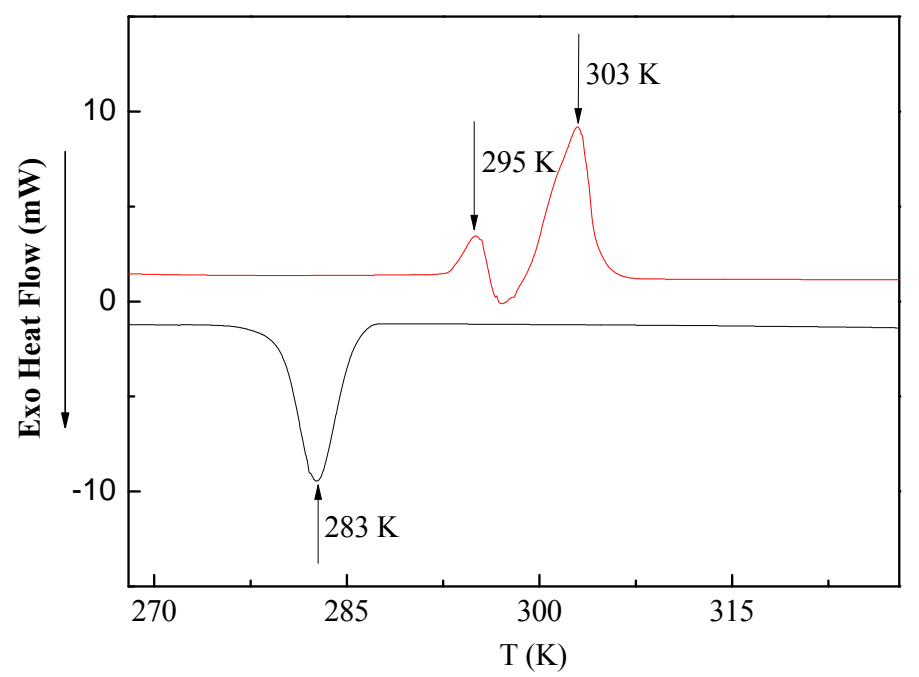

From Figure 3, it becomes clear that only one crystallization peak in the vicinity of $283 \mathrm{~K}$ during the cooling process was apparent. However, subsequent heating showed that there are unambiguously two melting peaks at $295 \mathrm{~K}$ and another at $303 \mathrm{~K}$. Similar multiple melting peak phenomena have been reported in studies with low molar mass PEO homopolymers. In those investigations with PEO, the untypical thermal behavior is known as the integral folding (IF) and nonintegral folding (NIF) structure. Kovacs et al. pioneered this issue and studied the relationship between linear growth rate, single crystal morphology and the IF structure [41-45]. Cheng et al. demonstrated the existence of the NIF structure using time resolved small-angle X-ray scattering (SAXS) [46-56]. At first glance, it seems that a similar mechanism might play a role for low molar mass PDMSB homopolymers, but further investigations are necessary, as described in the following section.

\subsection{XRD and SAXS Measurements of PDMSB Homopolymers}

In Figure 4, the X-ray diffraction (XRD) pattern of the PDMSB sample with an average molar mass of $5.6 \mathrm{~kg} \mathrm{~mol}^{-1}$ is shown, which has been isothermally crystallized at $283 \mathrm{~K}$ after annealing at $353 \mathrm{~K}$ for $10 \mathrm{~min}$. The scattering pattern consists of a series of narrow Bragg peaks. Obviously, the sample is partially crystalline. The peak positions are the same as those reported previously by Kawahara et al. [34]. Only the relative intensity of the peaks is different. This can be caused by a different orientation of crystallites in the samples as a consequence of the sample preparation procedure. 
Figure 4. X-ray diffraction pattern of PDMSB with an average molar mass of $5.6 \mathrm{~kg} \mathrm{~mol}^{-1}$ isothermally crystallized at $283 \mathrm{~K}$ after annealing at $353 \mathrm{~K}$ for $10 \mathrm{~min}$.

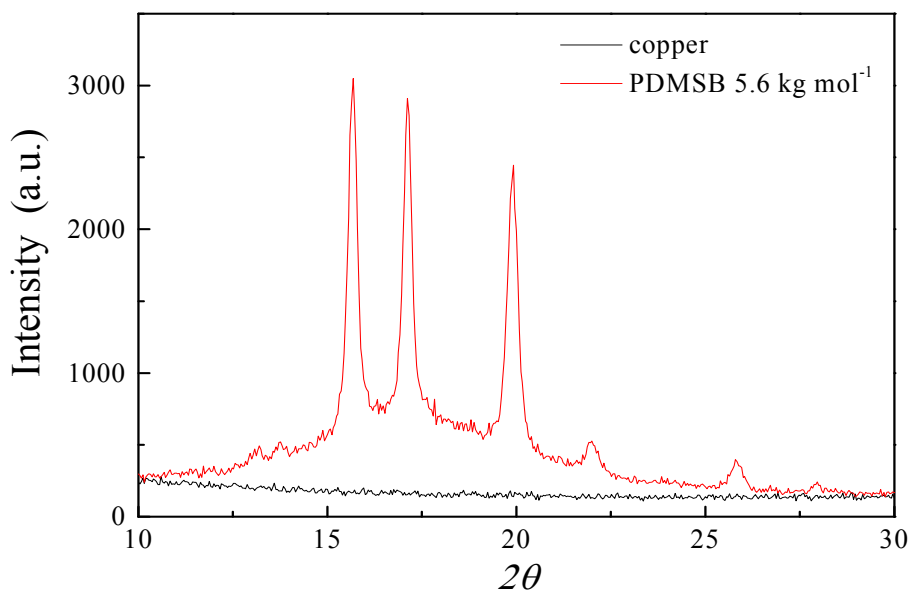

We now turn to the crystalline/amorphous mesostructure of the sample. Figure 5 shows the Lorentz-corrected SAXS profile for the melt-crystallized sample at $283 \mathrm{~K}$ for $120 \mathrm{~min}$. The results show one long range order peak, which relates to a long period of $10.7 \mathrm{~nm}$. This differs from observations in the isothermal crystallization behavior of PEO homopolymers [48]. For PEO, it has been found that at first, a scattering peak was observed during isothermal crystallization, followed by a decrease of the scattering peak, and the appearance of two other scattering peaks developed gradually. Isothermal thickening and/or thinning processes lead to the formation of the final IF chain crystals for low molecular weight PEO, which can be used to explain the multiple melting peaks phenomenon. However, for PDMSB, this is not the case, as only one scattering peak is observed after crystallization.

Figure 5. Lorentz-corrected small-angle X-ray scattering (SAXS) profile evaluated for the melt-crystallized sample at $283 \mathrm{~K}$ for $120 \mathrm{~min} . q_{m}{ }^{*}$ denotes the scattering vector corresponding to the reciprocal of the long period.

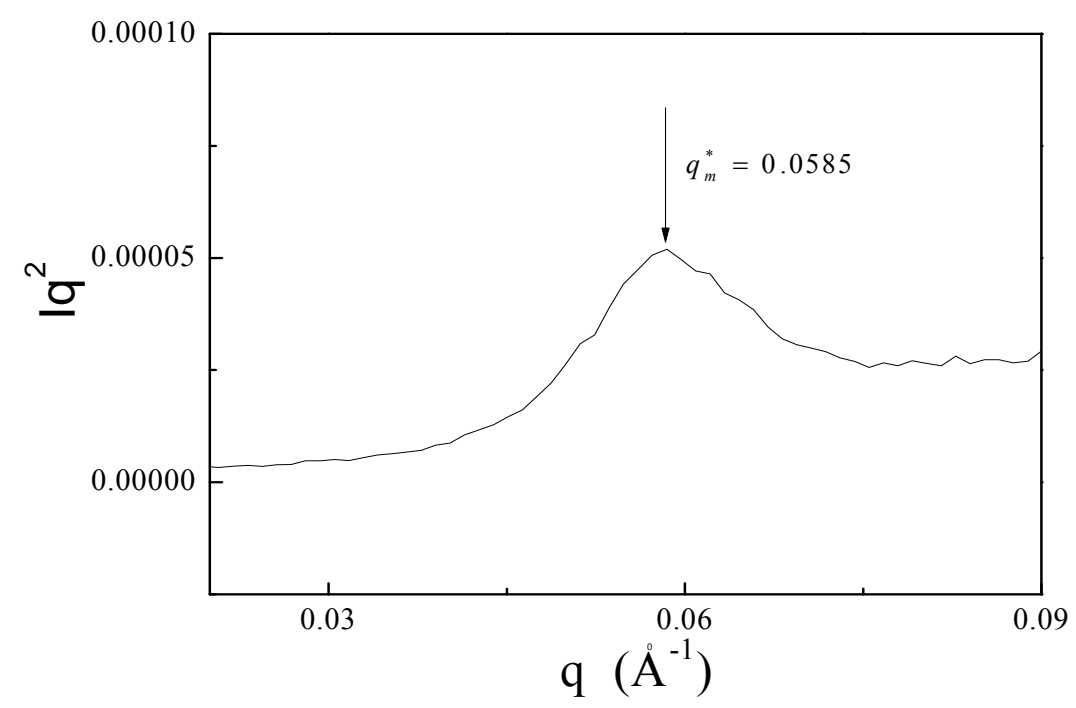


To sum up, for all results derived by DSC, XRD and SAXS measurements for low molar mass PDMSB homopolymers, we assume that the first melting peak corresponds to crystal lamellae formed during isothermal crystallization, while the second one is caused by recrystallization during the heating process. DSC measurements show that when isothermal crystallization temperature, $T_{c}$, is low enough, there will be two melting peaks, but only one melting peak when $T_{c}$ is high. Recent DSC curves of isothermal crystallized PDMSB samples also show that there is only one peak without any shoulders. This interesting crystallization behavior for low molar mass PDMSB will be explicitly discussed in a prospective work.

Investigations regarding the crystallization of PDMSB homopolymers with higher molar masses show a fast and distinct crystallization, which shows PDMSB to be an excellent candidate for studying the influence of the crystallization of anchored polymers on a curved substrate, as described in the following sections.

\subsection{Surface-Initiated Anionic Polymerization of DMSB on Cross-Linked PS Nanoparticles}

As a solid model substrate for the intended surface-initiated anionic polymerization of DMSB and to study the crystallization behavior of surface-attached PDMSB chains, PS nanoparticles were used, which were cross-linked with $10 \mathrm{wt} \%$ divinylbenzene. Those particles were used because of their simple synthesis via seeded emulsion polymerization, similar to a previously described procedure, leading to almost monodisperse particles [57]. For further purification, the particles were dried by three-fold azeotropic distillation with dry toluene under high vacuum conditions. To ensure the absence of protic impurities, which would immediately cause a termination of the anionic polymerization, the particle dispersion in toluene was additionally titrated, as described in the following. Therefore, sec-BuLi was slowly added with a $\mu \mathrm{L}$-syringe, until a slightly yellowish color appeared, which remained for at least $15 \mathrm{~min}$, due to the nucleophilic addition at the accessible vinyl groups in the swollen particles' shell. In the next step, the calculated amount of initiator for the intended molar masses of PDMSB was added, followed by DMSB monomer addition (Figure 6). The polymerization was terminated with degassed methanol, and surface-grafted particles were purified by repeated centrifugation and redispersing in neat THF for at least five times.

Figure 6. Surface-initiated anionic ROP of DMSB on the surface of PS nanoparticles.
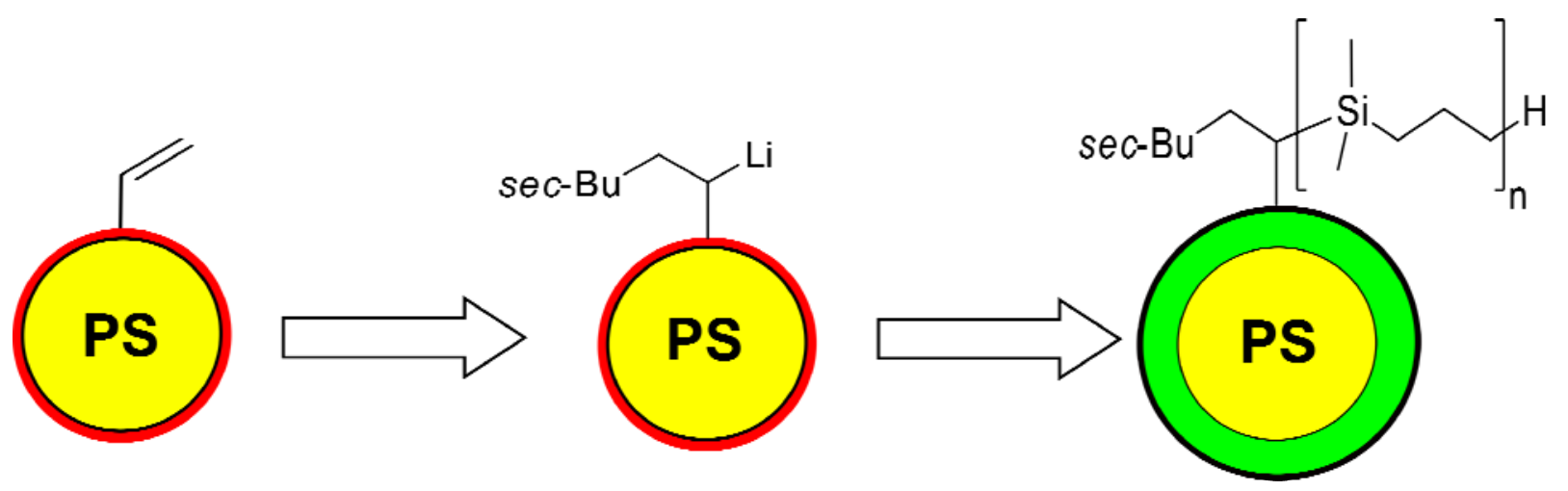


\subsection{Characterization of PDMSB-Grafted PS Nanoparticles}

\subsubsection{TEM Investigations}

Purified PDMSB-grafted PS particles were dispersed in neat THF and drop-casted on a carbon-coated copper grid for intended TEM investigations. The images shown in Figure 7 compared to the images of bare PS particles reveal that anionic surface-grafting was successful, due to the film formation during the sample preparation process caused by the particles' shell, which consisted of linear PDMSB chains. Gray scale analysis of PDMSB@PS-2 TEM images reveal a particle-particle distance of approx. $102 \mathrm{~nm}$, which corresponds to a shell thickness for each particle of $51 \mathrm{~nm}$ in the solid state.

Figure 7. TEM images of bare cross-linked PS particles (a) and surface-modified PDMSB particles with high content (b) and low content of PDMSB (c).
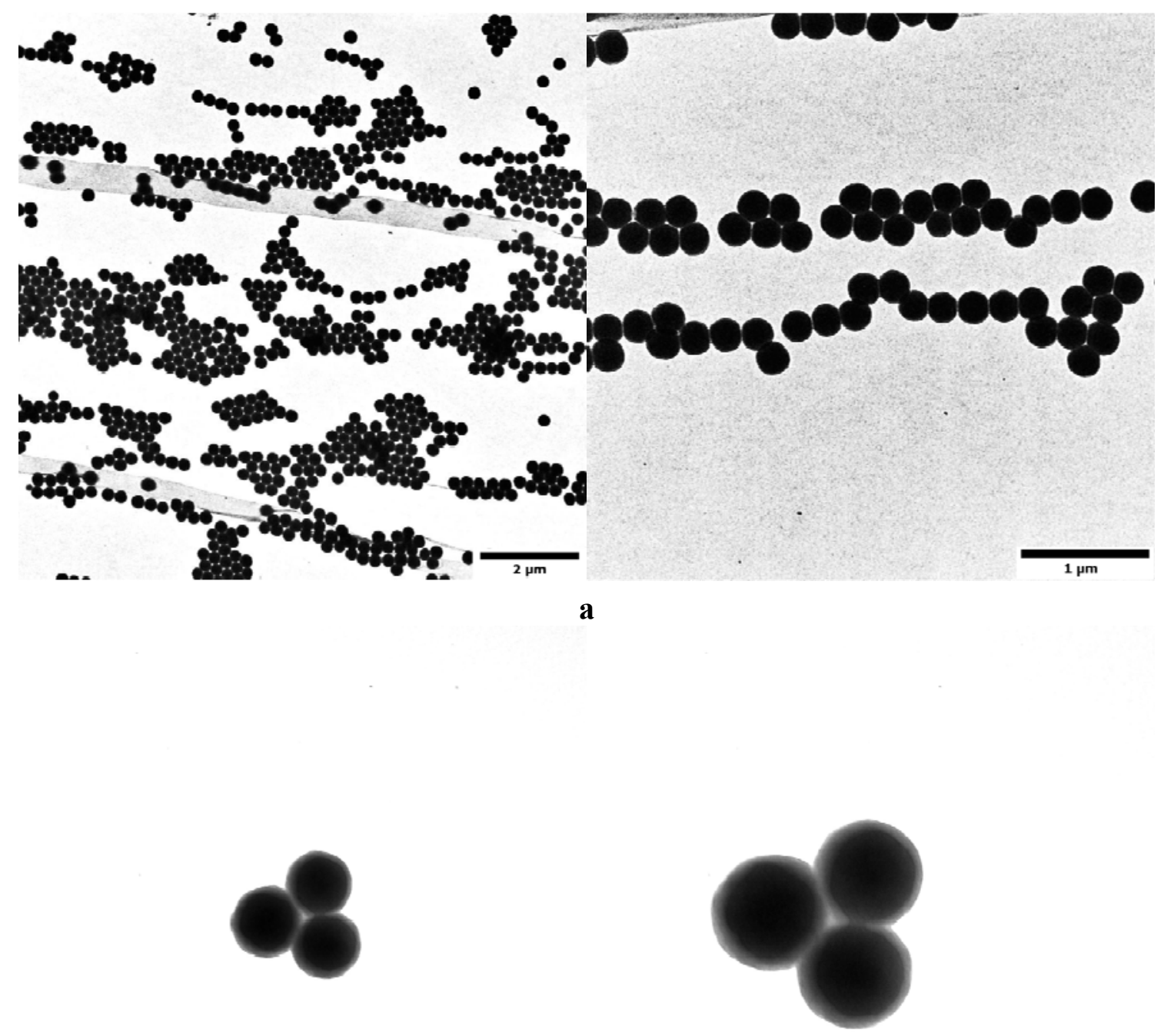
Figure 7. Cont.

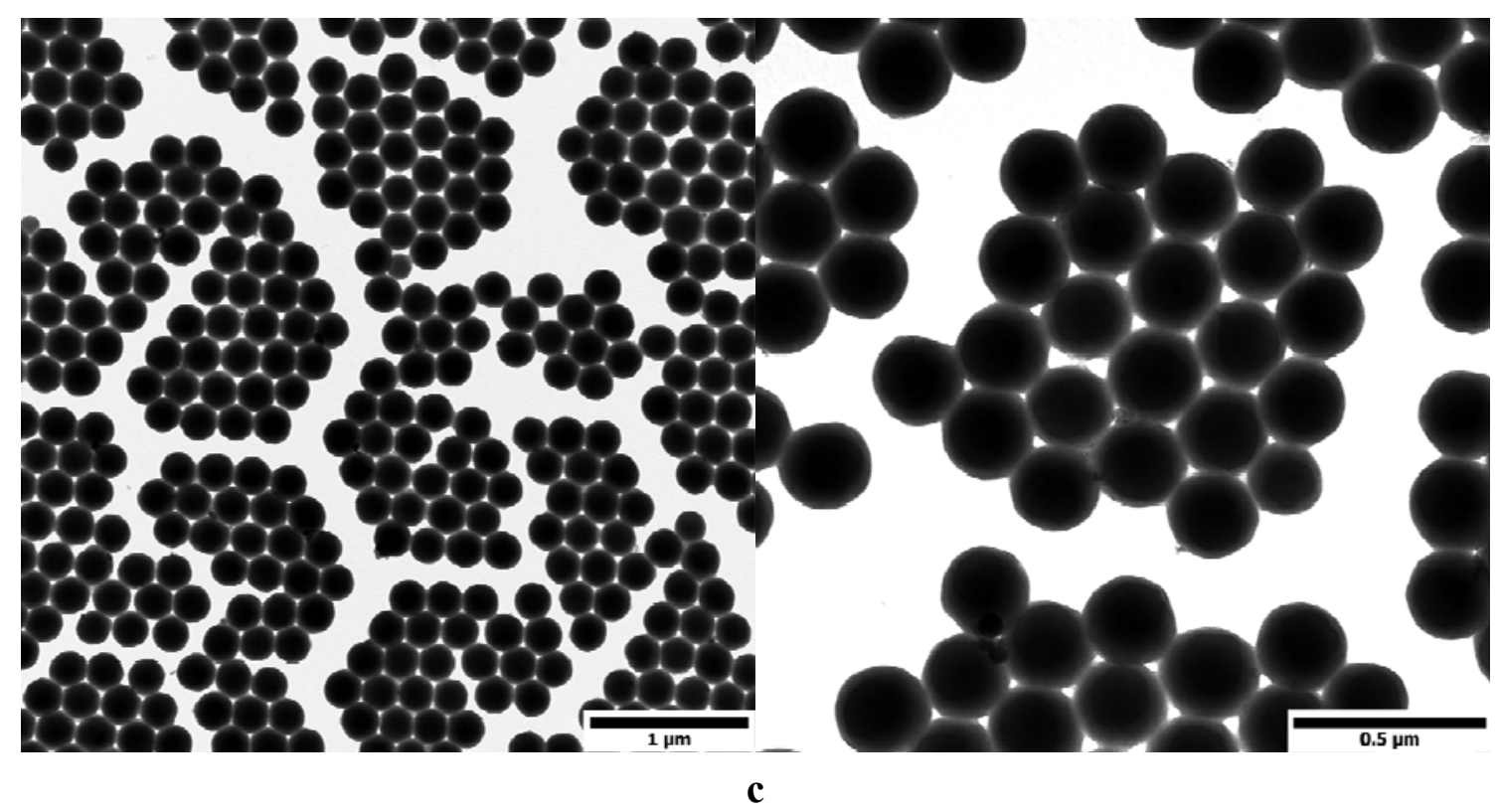

\subsubsection{DLS Measurements of PDMSB-Grafted PS Particles}

To prove the successful grafting-from approach for DMSB on the surface of PS particles additionally to TEM studies, DLS measurements were done to obtain changes of the hydrodynamic radii and diffusion coefficients of the grafted particles. In all cases, the correlation functions, $\mathrm{g}_{2}$, were found to be very close to exponential, and no indication of polydispersity was found. The correlation time, $\tau$, is related to the translational diffusion coefficient, $D$, by $D=1 /\left(\tau q^{2}\right)$, and the expected linear variation of $\langle\tau\rangle^{-1}$ with $q^{2}$ was very nicely fulfilled by our data. Figure 8 shows, therefore, the scattering vector $\left(q^{2}\right)$ dependence of the reciprocal average relaxation time $\langle\tau\rangle^{-1}$ for bare and grafted PS nanoparticles in THF. Applying the Stokes-Einstein relation allowed to calculate hydrodynamic radii, $\mathrm{R}_{\mathrm{h}}=k_{B} \mathrm{~T} / 6 \pi \eta \mathrm{D}$, with $k_{B}, \mathrm{~T}$ and $\eta$ as the Boltzmann constant, the absolute temperature and the solvent viscosity, respectively. From results in Figure 8, it can be concluded that the slope decreases after PDMSB-grafting of the PS nanoparticles and, hence, indicating an increase in hydrodynamic radii. Obtained $R_{h}$ increases from $148 \mathrm{~nm}$ for bare PS nanoparticles to $209 \mathrm{~nm}$ for sample PDMSB@PS-1 and to 175 nm for sample PDMSB@PS-2, which corresponds to a shell thickness of $61 \mathrm{~nm}$ for the first and $27 \mathrm{~nm}$ for the second sample, respectively, evidencing a successful particle grafting with PDMSB. 
Figure 8. Scattering vector $\left(q^{2}\right)$ dependence of the reciprocal average relaxation times, $<\tau>^{-1}$, for bare PS (black) and two different PDMSB-grafted PS particles (blue and red).

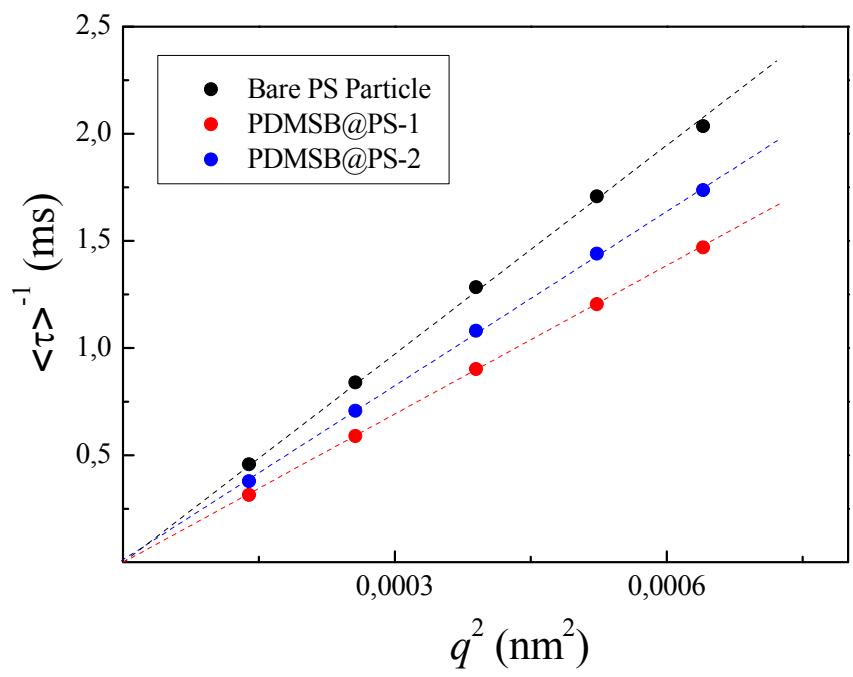

\subsubsection{DSC Investigations of PDMSB-Grafted PS Particles}

To gain first insights into the crystallization behavior of PDMSB chains attached on a curved substrate, the grafted particles were investigated by using DSC in a similar manner as the PDMSB homopolymers. PDMSB-grafted PS particles were heated to $473 \mathrm{~K}$, and the temperature was held for $10 \mathrm{~min}$ to remove the thermal history. The sample was measured with a cooling rate of $30 \mathrm{~K} \mathrm{~min}{ }^{-1}$ down to $253 \mathrm{~K}$, followed by heating again to $473 \mathrm{~K}$ with a rate of $30 \mathrm{~K} \mathrm{~min}^{-1}$. The results are given in Figure 9 for sample PS@PDMSB-1, the sample with a higher content of PDMSB attached on the surface of the particles, and in Figure 10, the DSC results are given for sample PS@PDMSB-2.

Figure 9. DSC diagram for sample PDMSB@PS-1 with a heat rate of $30 \mathrm{~K} \mathrm{~min}^{-1}$.

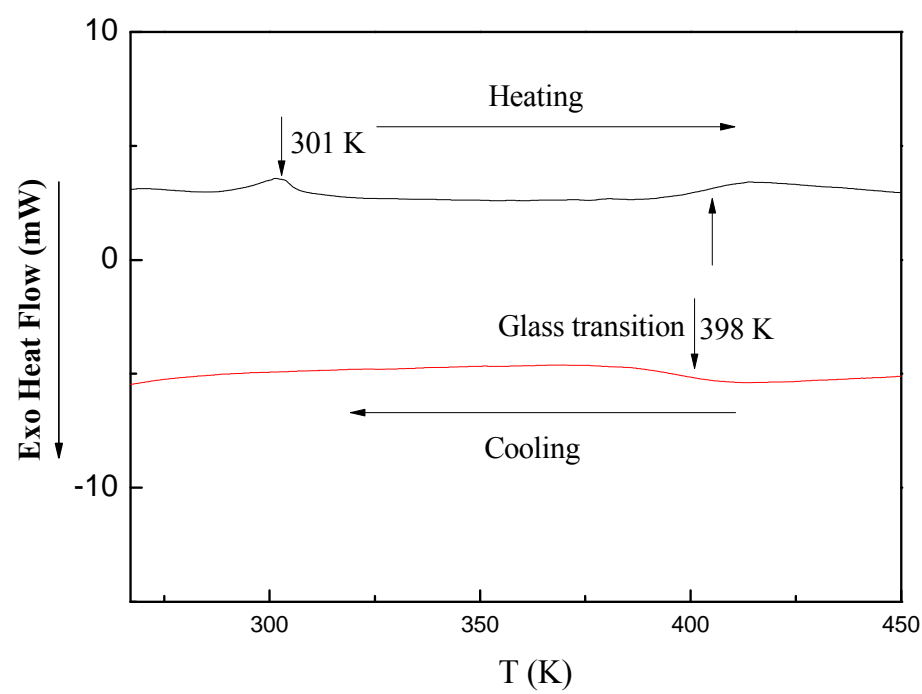


Figure 10. DSC diagram for sample PDMSB@PS-2 with a heat rate of $30 \mathrm{~K} \mathrm{~min}^{-1}$.

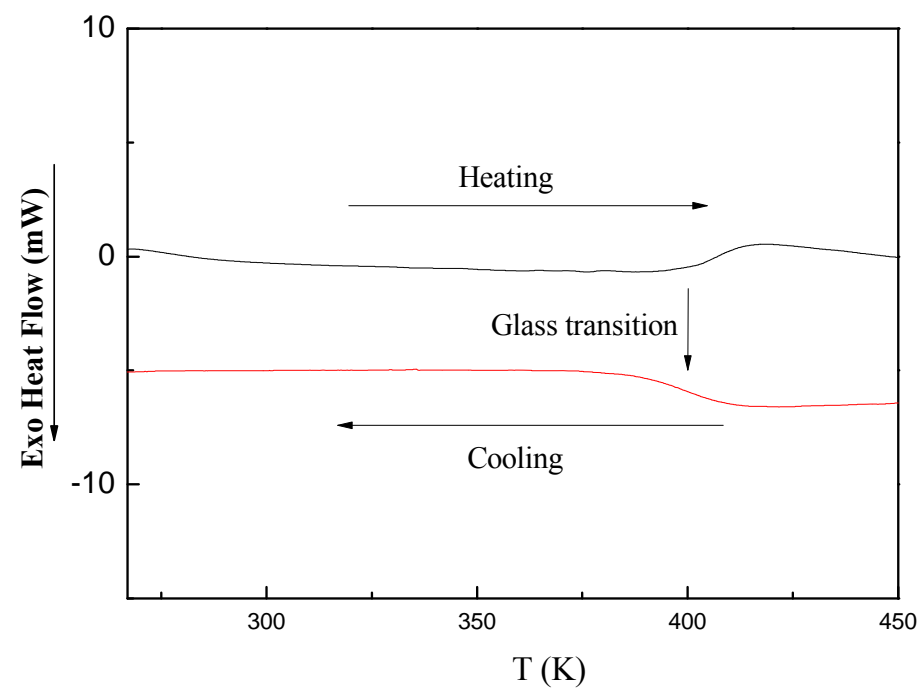

Both DSC diagrams in Figures 9 and 10 show the glass transition caused by the cross-linked PS nanoparticles. The transition temperature was estimated to be $398 \mathrm{~K}$, which is higher than the glass transition of PS homopolymers $(380 \mathrm{~K})$ due to the crosslinking of PS with DVB [58]. Also, during the cooling process, no crystallization peak could be observed, as is the case for PDMSB homopolymers, independently of obtained molar masses. This can be explained by either too few PDMSB chains attached at the surface of PS nanoparticles or a tremendous influence of the curved substrate both hampering the crystallization. In the subsequent heating process, a weak melting peak was observed at about $301 \mathrm{~K}$, which is very close to the melting peak of PDMSB homopolymers (303 K). In contrast to PDMSB homopolymer investigations, only one melting peak was observed. This is because the diffusion of PDMSB chains attached to the surface is remarkably hindered, and the chains can only crystallize locally at the surface of PS nanoparticles. Hence, the recrystallization process is inhibited. Figure 10 is the DSC diagram of PS@PDMSB-2. From DLS results in the previous section, $\mathrm{R}_{\mathrm{h}}$ for PS@PDMSB-2 could be determinated to $175 \mathrm{~nm}$, which was much smaller than the value of PS@PDMSB-2 (209 nm). This means that there is even less crystallizable material at the surface. And indeed, DSC results show that after sample treatment, to obtain the same thermal history, there is no melting peak during the heating process for PS@PDMSB-2.

\section{Conclusions}

In conclusion, a synthetic protocol leading to crystallizable poly(1,1-dimethylsilacyclobutane) (PDMSB) homopolymers with molar masses ranging from $2.3 \mathrm{~kg} \mathrm{~mol}^{-1}$ to $60 \mathrm{~kg} \mathrm{~mol}^{-1}$ with low polydispersities for every sample (PDI between 1.04 and 1.15) was reported. Absolute molar masses of investigated polycarbosilanes (PCS) were determinated by using SEC-MALLS and were directly compared to SEC measurements, with PS standards revealing a slightly lower molar mass for investigated samples in the latter case. Low molar mass PDMSB homopolymers were found to be rapidly crystallizing. They showed an interesting crystallization phenomenon, which at first glance, was similar to the untypical crystallization of low molar mass poly(ethylene oxide)s. However, SAXS, 
DSC and XRD studies revealed that crystallization behavior is different compared to low molar mass PEO, and recrystallization is assumed to be the reason for the appearance of two crystallization peaks. PDMSB was found to be an excellent candidate for studying the crystallization of anchored polymers influenced on a curved substrate. Therefore, the anionic ROP of DMSB was expanded to surface-initiated anionic polymerization on the surface of cross-linked PS nanoparticles. The resulting rigorously purified PCS-grafted nanoparticles were studied by using TEM and DLS measurements, evidencing the generation of novel PCS/organic core/shell particles. DSC results showed that crystallization of surface-attached PDMSB chains is hindered on the curved particles' surface. Prospective studies will focus on further characterizations of the untypical low molar mass PDMSB crystallization behavior and pyrolysis of the herein presented novel PCS/organic core/shell nanoparticles.

\section{Acknowledgments}

The authors would like to thank Marion Trautmann and Matthias Wittemann for SEC measurements and Frank Fischer for help with XRD measurements. The authors want to thank the Deutsche Forschungsgemeinschaft DFG-SPP 1369 (Interfaces and Interphases RE 923/14-2 and STU 191/3-2) and the Landesoffensive zur Entwicklung Wissenschaftlich-ökonomischer Exzellenz (LOEWE Soft Control) for financial support of this work.

\section{References}

1. Birot, M.; Pillot, J.-P.; Dunogues, J. Comprehensive chemistry of polycarbosilanes, polysilazanes, and polycarbosilazanes as precursors of ceramics. Chem. Rev. 1995, 95, 1443-1477.

2. Sanchez, J.C.; Trogler, W.C. Hydrosilylation of diynes as a route to functional polymers delocalized through silicon. Macromol. Chem. Phys. 2008, 209, 1527-1540.

3. Vakifahmetoglu, C. Fabrication and properties of ceramic 1D nanostructures from preceramic polymers: a review. Adv. Appl. Ceram. 2011, 110, 188-204.

4. Fukushima, M.; Colombo, P. Silicon carbide-based foams from direct blowing of polycarbosilane. J. Eur. Ceram. Soc. 2012, 32, 503-510.

5. Schawaller, D.; Clauß, B.; Buchmeiser, M.R. Ceramic filament fibers-a review. Macromol. Mater. Eng. 2012, 297, 502-522.

6. Yamashita, H.; Suzuki, Y.; Rao, T.V.; Uchimaru, Y. Synthesis and electrochemical, optical, and thermal properties of polycarbosilanes with silylene-vinylene-phenylene-vinylene backbones and triphenylamine or carbazole unit-containing side chains. J. Organomet. Chem. 2012, 710, 59-67.

7. Gupta, R.K.; Mishra, R.; Tiwari, R.K.; Ranjan, A.; Saxena, A.K. Studies on the rheological behavior of polycarbosilane part i: Effect of time, temperature and atmosphere. silicon 2010, 3, $27-35$.

8. Vakifahmetoglu, C.; Balliana, M.; Colombo, P. Ceramic foams and micro-beads from emulsions of a preceramic polymer. J. Eur. Ceram. Soc. 2011, 31, 1481-1490.

9. Friebe, L.; Liu, K.; Obermeier, B.; Petrov, S.; Dube, P.; Manners, I. Pyrolysis of Polycarbosilanes with pendant nickel clusters: Synthesis and characterization of magnetic ceramics containing nickel and nickel silicide nanoparticles. Chem. Mater. 2007, 19, 2630-2640. 
10. Chen, J.; He, G.; Liao, Z.; Zeng, B.; Ye, J.; Chen, L.; Xia, H.; Zhang, L. Control of structure formation of polycarbosilane synthesized from polydimethylsilane by Kumada rearrangement. J. Appl. Polym. Sci. 2008, 108, 3114-3121.

11. Fang, Y.; Huang, M.; Yu, Z.; Xia, H.; Chen, L.; Zhang, Y.; Zhang, L. Synthesis, Characterization, and Pyrolytic Conversion of a Novel Liquid Polycarbosilane. J. Am. Ceram. Soc. 2008, 91, 3298-3302.

12. Li, H.; Zhang, L.; Cheng, L.; Wang, Y.; Yu, Z.; Huang, M.; Tu, H.; Xia, H. Polymer-ceramic conversion of a highly branched liquid polycarbosilane for SiC-based ceramics. J. Mater. Sci. 2008, 43, 2806-2811.

13. Yu, Z.; Li, R.; Zhan, J.; Zhou, C.; Yang, L.; He, G.; Xia, H. Synthesis and characterization of a propargyl-substituted polycarbosilane with high ceramic yield. J. Appl. Polym. Sci. 2011, 121, 3400-3406.

14. Will, U.; Veljanovski, D.; Harter, P.; Rieger, B. Hyperbranched Polycarbosilanes of Homogeneous Architecture: Regioselective Hydrosilylation of AB2Monomers and Consecutive Functionalization. Macromolecules 2010, 43, 934-938.

15. Liao, C.X.; Weber, W.P. Synthesis and Properties of Novel Functionally Substituted Carbosilane Polymers. Macromolecules 1993, 26, 563-566.

16. Ganicz, T.; Staficzyk, W.; Biatecka-Florjaficzyk, E.; Sledzifiska, I. Synthesis and characterization of liquid crystal polycarbosilanes: poly(1-methyl-1-silaethylene), poly(1-methyl-1-silabutane)and poly(1-silabutane) with pendant mesogenic groups. Polymer 1996, 37, 4167-4174.

17. Shankar, R.; Saxena, A.; Brar, A.S. Synthesis and reactivity of novel oligosilanes bearing functional carbosilane side chains. J. Organomet. Chem. 2001, 628, 262-270.

18. Wurm, F.; Schule, H.; Frey, H. Amphiphilic Linear-Hyperbranched Block Copolymers with Linear Poly(ethylene oxide) and Hyperbranched Poly(carbosilane) Block. Macromolecules 2008, 41, 9602-9611.

19. Wurm, F.; Hilf, S.; Frey, H. Electroactive linear-hyperbranched block copolymers based on linear poly(ferrocenylsilane)s and hyperbranched poly(carbosilane)s. Chem. Eur. J. 2009, 15, 9068-9077.

20. Kudo, H.; Fujiwara, Y.; Miyasaka, M.; Nishikubo, T. Synthesis of polycarbosilanes by A2 + Bn $(\mathrm{n}=2,3$, and 4$)$ type hydrosilylation reaction and evaluation of their refractive index properties. $J$. Polym. Sci. A Polym. Chem. 2010, 48, 5746-5751.

21. Schacher, F.H.; Rupar, P.A.; Manners, I. Functional Block Copolymers: Nanostructured Materials with Emerging Applications. Angew. Chem. Int. Ed. 2012, 51, 7898-7921.

22. Rangou, S.; Shishatskiy, S.; Filiz, V.; Abetz, V. Poly(vinyl trimethylsilane) and block copolymers of vinyl trimethylsilane with isoprene: Anionic polymerization, morphology and gas transport properties. Eur. Polym. J. 2011, 47, 723-729.

23. Zhou, S.Q.; Park, Y.T.; Manuel, G.; Weber, W.P. Anionic ring opening polymerization of 1-silacyclopent-3-ene. Polym. Bull. 1990, 23, 491-496.

24. Liao, C.X.; Weber, W.P. Synthesis and characterization of poly(1-methyl-1-silabutane), poly(1-phenyl-1-silabutane) and poly(1-silabutane). Polym. Bull. 1992, 28, 281-286.

25. Theurig, M.; Weber, W.P. Stereoselective anionic ring opening polymerization of 1,1-dimethyl-1silacyclobutene. Polym. Bull. 1992, 28, 17-21. 
26. Rulkens, R.; Lough, A.J.; Manners, I. Anionic Ring-Opening Oligomerization and Polymerization of Silicon-Bridged [1]Ferrocenophanes: Characterization of Short-Chain Models for Poly(ferrocenylsilane) High Polymers. J. Am. Chem. Soc. 1994, 116, 797-798.

27. Suzuki, M.; Kotani, J.; Gyobu, S.; Kaneko, T.; Saegusa, T. Synthesis of Sequence-Ordered Polysilane by Anionic Ring-Opening Polymerization of Phenylnonamethylcyclopentasilane. Macromolecules 1994, 27, 2360-2363.

28. Kulbaba, K.; Manners, I. Polyferrocenylsilanes: Metal-Containing Polymers for Materials Science, Self-Assembly and Nanostructure Applications. Macromol. Rap. Comm. 2001, 22, 711-724.

29. Bellas, V.; Rehahn, M. Polyferrocenylsilan-basierte Polymersysteme. Angew. Chem. 2007, 119, 5174-5197.

30. Bellas, V.; Rehahn, M. Polyferrocenylsilane-Based Polymer Systems. Angew. Chem. Int. Ed. 2007, 46, 5082-5104.

31. Knischka, R.; Frey, H.; Rapp, U.; Mayer-Posner, F.J. Living anionic ring-opening polymerization of 1,1-dipropylsilacyclobutane. Macromol. Rap. Comm. 1998, 19, 455-459.

32. Matsumoto, K.; Yamaoka, H. Living Anionic Ring-Opening Polymerization of 1,1-Dimethylsilacyclobutane. Macromolecules 1995, 28, 7029-7031.

33. Matsumoto, K.; Shimazu, H.; Deguchi, M.; Yamaoka, H. Anionic Ring-Opening Polymerization of Silacyclobutane Derivatives. J. Polym. Sci. A Poly. Chem. 1997, 35, 3207-3216.

34. Kawahara, S.; Nagai, A.; Kazama, T.; Takano, A.; Isono, Y. Preparation of Poly(1,1-dimethyl silabutane) by Anionic Polymerization and Its Crystallization. Macromolecules 2004, 37, 315-321.

35. Nghiem, Q.D.; Kim, D.-P. Direct Preparation of High Surface Area Mesoporous SiC-Based Ceramic by Pyrolysis of a Self-Assembled Polycarbosilane-block-Polystyrene Diblock Copolymer. Chem. Mater. 2008, 20, 3735-3739.

36. Gallei, M.; Tockner, S.; Klein, R.; Rehahn, M. Silacyclobutane-Based Diblock Copolymers with Vinylferrocene, Ferrocenylmethyl Methacrylate, and [1]Dimethylsilaferrocenophane. Macromol. Rap. Comm. 2010, 31, 889-896.

37. Ungar, G.; Putra, E.G. R.; de Silva, D.S. M.; Shcherbina, M.A.; Waddon, A.J. The effect of self-poisoning on crystal morphology and growth rates. Adv. Polym. Sci. 2005, 180, $45-87$.

38. Keller, N.; Pham-Huu, C.; Crouzet, C.; Ledoux, M.J.; Savin-Poncet, S.; Nougayrede, J.-B.; Bousquet, J. Direct oxidation of H2S into S. New catalysts and processes based on SiC support. Catal. Today 1999, 53, 535-542.

39. Connolly, E.J.; O'Halloran, G.M.; Pham, H.T.M.; Sarro, P.M.; French, P.J. Comparison of porous silicon, porous polysilicon and porous silicon carbide as materials for humidity sensing applications. Sens. Actuators A 2002, 99, 25-30.

40. TamilSelvan, S.; Aldeyab, S.S.; Zaidi, J.S. M.; Arivuoli, D.; Ariga, K.; Mori, T.; Vinu, A. Preparation and characterization of highly ordered mesoporous SiC nanoparticles with rod shaped morphology and tunable pore diameters. Journal of Materials Chemistry 2011, 21, 8792-8799.

41. Kovacs, A.J.; Gonthier, A. Crystallization and fusion of self-seeded polymers. Colloid Polym. Sci. 1972, 250, 530-551. 
42. Kovacs, A.J.; Gonthier, A.; Straupe, C. Isothermal growth, thickening, and melting of poly(ethylene oxide) singel crystals in the bulk. J. Polym. Sci. Polym. Symp. 1975, 50, 283-325.

43. Kovacs, A.J.; Straupe, C.; Gonthier, A. Isothermal growth, thickening, and melting of poly(ethylene oxide) singel crystals in the bulk. J. Polym. Sci. Polym. Symp. 1977, 59, 31-54.

44. Kovacs, A.J.; Straupe, C. Isothermal growth, thickening and melting of poly(ethylene oxide) single crystals in the bulk. Part 4: Dependence of pathological crystal habits on temperature and thermal history. Faraday Discuss. Chem. Soc. 1979, 68, 225-239.

45. Kovacs, A.J.; Straupe, C. Isothermal growth, thickening and melting of poly(ethylene oxide) single crystals in the bulk. J. Cryst. Growth 1980, 48, 210-226.

46. Cheng, S.Z. D.; Zhang, A.; Chen, J. Existence of a Transient Nonintegral Folding Lamellar Crystal in a Low Molecular Mass Poly (Ethylene Oxide) Fraction Crystallized from the Melt. $J$. Polym. Sci. C Polym. Lett. 1990, 28, 233-239.

47. Cheng, S.Z. D.; Zhang, A.; Barley, J.S.; Chen, J.; Habenschuss, A.; Zschack, P.R. Isothermal Thickening and Thinning Processes in Low Molecular Weight Poly(ethylene oxide) Fractions. 1. From Nonintegral-Folding to Integral-Folding Chain Crystal Transitions. Macromolecules 1991, 24, 3973-3944.

48. Cheng, S.Z.D.; Zhang, A.; Chen, J.; Heberer, D.P. Nonintegral and Integral Folding Crystal Growth in Low-Molecular Mass Poly (ethylene Oxide) Fractions. I. Isothermal lamellar Thickening and Thinning. J. Polym. Sci. B Polym. Phys. 1991, 29, 287-297.

49. Cheng, S.Z.D.; Chen, J.; Zhang, A.; Heberer, D.P. Nonintegral and Integral Folding Crystal Growth in Low-Molecular Mass Poly (ethylene Oxide) Fractions. II. End-Group Effect:

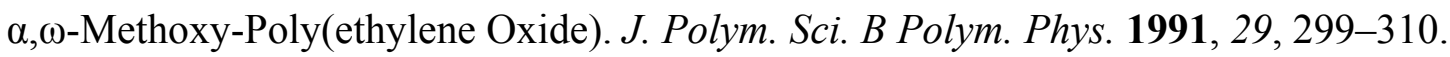

50. Cheng, S.Z.D.; Chen, J. Nonintegral and Integral Folding Crystal Growth in Low-Molecular Mass Poly (ethylene Oxide) Fractions,III. Linear Crystal Growth Rates and Crystal Morphology. $J$. Polym. Sci. B Polym. Phys. 1991, 29, 311-327.

51. Cheng, S.Z.D.; Chen, J.; Barley, J.S.; Zhang, A. Isothermal Thickening and Thinning Processes in Low Molecular Weight Poly(ethylene oxide) Fractions Crystallized from the Melt.3. Molecular Weight Dependence. Macromolecules 1992, 25, 1453-1460.

52. Cheng, S.Z.D.; Wu, S.S.; Chen, J.; Zhuo, Q.; Quirk, R.P.; von Meerwall, E.D.; Hsiao, B.S.; Habenschuss, A.; Zschack, P.R. Isothermal Thickening and Thinning Processes in Low Molecular Weight Poly(ethylene oxide) Fractions Crystallized from the Melt.4. End-Group Dependence. Macromolecules 1993, 26, 5105-5117.

53. Cheng, S.Z.D.; Chen, J.; Wu, S.X.; Zhang, A.; Yandrasits, M.A.; Zhuo, Q.; Quirk, R.P.; Habenschuss, A.; Zschack, P.R. Nonintegral and Integral Folding Crystal Growth in Low Molecular Weight Poly(Ethylene Oxide) Fractions. Cryst. Polym. 1993, 405, 51-62.

54. Lee, S.-W.; Chen, E.; Zhang, A.; Yoon, Y.; Moon, B.S.; Lee, S.; Harris, F.W.; Cheng, S.Z. D. Isothermal Thickening and Thinning Processes in Low Molecular Weight Poly(ethylene oxide) Fractions Crystallized from the Melt. 5. Effect of Chain Defects. Macromolecules 1996, 29, 8816-8823.

55. Chen, E.-Q.; Lee, S.-W.; Zhang, A.; Moon, B.-S.; Mann, I.; Harris, F.W.; Cheng, S.Z. D.; Hsiao, B.S.; Yeh, F.; Merrewell, E.von; Grubb, D.T. Isothermal Thickening and Thinning Processes in 
Low-Molecular-Weight Poly(ethylene oxide) Fractions Crystallized from the Melt. 8. Molecular Shape Dependence. Macromolecules 1999, 32, 4784-4793.

56. Chen, E.-Q.; Lee, S.-W.; Zhang, A.; Moon, B.-S.; Honigfort, P.S.; Mann, I.; Lin, H.-M.; Harris, F.W.; Cheng, S.Z. D.; Hsiao, B.S.; Yeh, F. Isothermal thickening and thinning processes in low molecular weight poly(ethylene oxide) fractions crystallized from the melt 6 . Configurational defects in molecules. Polymer 1999, 40, 4543-4551.

57. Mazurowski, M.; Gallei, M.; Li, J.; Didzoleit, H.; Stühn, B.; Rehahn, M. Redox-Responsive Polymer Brushes Grafted from Polystyrene Nanoparticles by Means of Surface Initiated Atom Transfer Radical Polymerization. Macromolecules 2012, 45, 8970-8981.

58. Rieger, J. The Glass Transition Temperature of Polystyrene. J. Therm. Anal. 1996, 46, 965-972.

(C) 2013 by the authors; licensee MDPI, Basel, Switzerland. This article is an open access article distributed under the terms and conditions of the Creative Commons Attribution license (http://creativecommons.org/licenses/by/3.0/). 